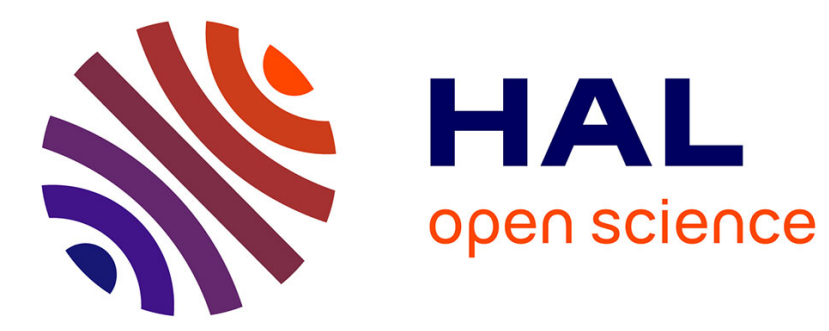

\title{
Hypothesis and Convention in Poincaré's Defense of Galilei Spacetime
}

\author{
Scott Walter
}

\section{To cite this version:}

Scott Walter. Hypothesis and Convention in Poincaré's Defense of Galilei Spacetime. Michael Heidelberger, Gregor Schiemann. The Significance of the Hypothetical in the Natural Sciences, Walter de Gruyter, pp.193-219, 2009, 10.1515/9783110210620.193 . halshs-01234431

\section{HAL Id: halshs-01234431 \\ https://shs.hal.science/halshs-01234431}

Submitted on 26 Nov 2015

HAL is a multi-disciplinary open access archive for the deposit and dissemination of scientific research documents, whether they are published or not. The documents may come from teaching and research institutions in France or abroad, or from public or private research centers.
L'archive ouverte pluridisciplinaire HAL, est destinée au dépôt et à la diffusion de documents scientifiques de niveau recherche, publiés ou non, émanant des établissements d'enseignement et de recherche français ou étrangers, des laboratoires publics ou privés. 


\title{
Hypothesis and Convention in Poincaré's Defense of Galilei Spacetime
}

\author{
Scott Walter* \\ The Significance of the Hypothetical in the Natural \\ Sciences, edited by Michael Heidelberger and Gregor \\ Schiemann, Berlin: Walter de Gruyter, 2009, 193-219
}

\begin{abstract}
According to the conventionalist doctrine of space elaborated by the French philosopher-scientist Henri Poincaré in the 1890 s, the geometry of physical space is a matter of definition, not of fact. Poincaré's Hertz-inspired view of the role of hypothesis in science guided his interpretation of the theory of relativity (1905), which he found to be in violation of the axiom of free mobility of invariable solids. In a quixotic effort to save the Euclidean geometry that relied on this axiom, Poincaré extended the purview of his doctrine of space to cover both space and time. The centerpiece of this new doctrine is what he called the "principle of physical relativity," which holds the laws of mechanics to be covariant with respect to a certain group of transformations. For Poincaré, the invariance group of classical mechanics defined physical space and time (Galilei spacetime), but he admitted that one could also define physical space and time in virtue of the invariance group of relativistic mechanics (Minkowski spacetime). Either way, physical space and time are the result of a convention.
\end{abstract}

\section{Introduction}

Perhaps more than any other figure in contemporary science, Henri Poincaré focused the attention of both philosophers and scientists on the role played by hypothesis in the pursuit of scientific knowledge. The history of twentieth-century philosophy of science is marked by his conventionalist philosophy of geometry, which troubled philosophers from Ernst Cassirer, Moritz Schlick and Hans Reichenbach in the 1910s and 1920s, Philipp Frank, Ernest Nagel and Adolf Grünbaum in the 1950s and 1960s, Lawrence Sklar, Hilary Putnam, David Malament, Michael Friedman and others from the 1970s and 1980s

*scott.walter [at] univ-lorraine.fr, University of Lorraine \& Henri-Poincaré Archives (CNRS, UMR 7117) 
up to the present ${ }^{1}$ Much of this philosophical discussion is concerned with the conventionality of simultaneity in relativity theory, a problem distinct, on one hand, from that of the conventionality of simultaneity in classical mechanics (first discussed by Poincaré in 1898), and on the other hand, from that of the Riemann-Helmholtz-Lie problem of space, which occupied Poincaré and his contemporaries in the late nineteenth century. At the end of his life, Poincaré fused these two problem sets, and realized the overriding necessity of a spacetime convention for the foundations of physics. The following account of Poincaré's progress toward the latter view proceeds chronologically, beginning with the elaboration of his doctrine of physical space (1880-1900), followed by a discussion of Poincaré's understanding of the hypothetical basis of the theory of relativity (1900-1906), and an analysis of the 1912 lecture "L'espace et le temps," in which Poincaré affirmed the central role of hypothesis and convention in the production of scientific knowledge.

\section{Poincaré's doctrine of physical space}

Poincaré's philosophy of geometry first took form following French debates in the 1870s over the logical coherence and physical meaning of non-Euclidean geometry. While no French mathematician had been directly involved in the reevaluation of the foundations of geometry of the 1820s and 1830s, the ideas advanced by Bernhard Riemann, Eugenio Beltrami, and Hermann Helmholtz found both partisans and opponents in late nineteenth-century France.

By 1869 at the latest, the French mathematical establishment had recognized the existence of non-Euclidean geometries. In that year the French Academy of Sciences published for the very last time a note purporting to prove the parallel postulate. Its author, Joseph Bertrand, held the chair of general and mathematical physics at the Collège de France and was also Professor of Analysis at the École polytechnique. Bertrand published a demonstration of the parallel postulate over the protests of fellow members of the geometry section, but the ensuing public scandal eventually led him to admit that the proof had met with less than universal approval (Pont 1986, 637).

Debate over the status of non-Euclidean geometry continued well after 1870, particularly among French philosophers. Paul Tannery's empiricism met with opposition from Neokantians Charles Renouvier and Louis Couturat, whose pet claim was that only Euclidean geometry could be objective, because it was the only geometry subtended by spatial intuition. Euclidean geometry was thereby an ideal science, and an example of synthetic a priori knowledge in the Kantian scheme. ${ }^{2}$

It was also in the 1870s that France's most brilliant mathematician came of age. Henri Poincaré (1854-1912) was educated at the École polytechnique, under the tutelage of Charles Hermite, Henri

\footnotetext{
${ }^{1}$ For a comprehensive overview, see Ben-Menahem (2006).

${ }^{2}$ On the reception of non-Euclidean geometry among francophone Neokantians, see Panza (1995).
} 
Résal, and Alfred Cornu, and at the École des mines, where Henry Le Chatelier taught chemistry. In 1879 he defended a doctoral thesis supervised by Gaston Darboux on the geometric theory of partial differential equations, and after a short stint as a mine inspector in northeastern France, was engaged to teach mathematics at the University of Caen (Rollet 2001).

Less than a year after arriving in Caen, Poincare entered the competition for the grand prize in mathematical sciences organized by the French Academy of Sciences, which required contestants to "perfect an important element of the theory of linear differential equations in one independent variable." His submission did not win the prize, even though the three supplements to his prize essay established a new class of automorphic functions, that Poincaré called "Fuchsian" functions, in honor of the German mathematician Lazarus Fuchs.

The connections between Fuchsian functions and conventionalist philosophy of geometry are numerous, as Zahar (1997) shows. What Poincaré's supplements reveal is that as early as 1880 , Poincaré understood geometry in terms of groups of transformations. Fuchsian functions, Poincaré discovered, are invariant under a certain class of linear transformations that form a group. The study of the group in question reduces to that of the translation group of hyperbolic geometry, prompting the young Poincaré to ask:

Just what is, in fact, a geometry? It is the study of the group of operations formed by the displacements a figure can go through without deformation. In Euclidean geometry this group reduces to rotations and translations. In Lobachevsky's pseudogeometry it is more complicated. (Poincaré 1997, 35)

Geometry is reduced here to group theory, in the spirit of Felix Klein's Erlanger Program, although it appears unlikely that Poincaré had any knowledge of this program at the time. Other printed sources on nonEuclidean geometry were available to him, including French translations of Beltrami and Helmholtz, and during his student days in the late 1870s, Poincaré may well have picked up from his teachers (Hermite, Darboux, Jordan) the idea that the motions of a rigid body form a group. There is a strong resemblance between Poincaré's presentation of his model of hyperbolic geometry and that of Beltrami. Both are circle models, differential-geometric, and refer to Lobachevsky alone, and although Poincaré does not mention Beltrami by name, the latter's work was surely an inspiration to him (Gray \& Walter 1997b).

Poincaré's prodigious discovery of Fuchsian functions propelled him into the higher echelons of French mathematics. In 1886 he became a full professor of mathematical physics and probability calculus at the Sorbonne (replacing Gabriel Lippmann), and was elected President of the French Mathematical Society. The next year he was selected to replace Edmond Laguerre in the geometry section of the Academy of Sciences at the Institut de France.

Once a member of the Institut, Poincaré published his first essay on the foundations of geometry. Strongly influenced by Sophus Lie's 
writings, the paper concludes with a reflection on the relation between geometry and the physical space of experience:

[I]n nature there exist remarkable bodies called solids and experience teaches us that the diverse motions these bodies can perform are related very closely to the diverse operations of the [Euclid] group. [...]

Thus the fundamental hypotheses of geometry are not experimental facts, and yet it is the observation of certain physical phenomena that picks them out from all possible hypotheses. (Poincaré 1887, 91, original emphasis)

Geometry is then essentially an abstract science, being the study of groups of transformations. Observation of displaced solids suggests the transformations of one particular geometry, corresponding to the Euclid group. Or as Poincare put it in a subsequent paper, our experience "played but a single role: it served as an occasion" (Poincaré 1903, 424).

While Poincarés stance on the formal nature of geometry is unambiguous in his 1887 essay, he does not reflect on the epistemological status of the geometry of physical space. The latter topic is first evoked in Poincaré's next essay on the foundations of geometry, which appeared Louis Olivier's Revue générale des sciences pures et appliquées, and reached a wide readership both in French and English, thanks to a translation published in Nature $3^{3}$

In the essay "Non-Euclidean geometries," Poincaré presents his conventionalist view of the foundations of geometry and physics. Reiterating his belief in the abstract nature of geometry, Poincare explains why geometry can not be an experimental science:

If geometry were an experimental science, it would not be an exact science, it would be subject to incessant revision. And that is not all: it would even today be shown to be erroneous, since we know that rigorously invariable solids do not exist. (Poincaré 1891, 773)

If we were to adopt the empiricist approach to geometry, Poincaré tells his readers in the latter passage, we would eliminate Euclidean geometry straightaway as a candidate geometry for physical space, since there are no perfectly-rigid solids in this space.

When Poincaré points out this conflict between the motion of solids and Euclidean geometry, he bruises our confidence in the truth of the geometric axioms. Worse news is yet to come, however, as Poincaré goes on to tell us that the axioms have no truth-value at all:

The geometrical axioms are then neither synthetic a priori judgments nor experimental facts. They are conventions; our choice, out of all possible conventions, is guided by experimental facts; but it remains free and is limited only by the necessity of avoiding all contradiction. [...] In other words, the axioms of geometry [...] are but definitions in disguise. This being so,

${ }^{3}$ Poincaré $(1891,1892 a)$. On Poincaré's collaboration with the periodicals edited by Louis Olivier and Xavier Léon see Rollet (2001). 
what should one think of the question: "Is Euclidean geometry true?" It is meaningless. (Poincaré 1891, 773, original emphasis)

In other words, as Nabonnand (2000) observes, inasmuch as Euclidean geometry is an abstract science, the truth of its theorems may not be ascertained by empirical means.

The same is true for the axioms of non-Euclidean geometry, and to argue the point, Poincaré asks what would happen if the parallax of a given star were observed to have a negative value (corresponding to elliptic space), or if all parallaxes were observed to be greater than a certain positive value (corresponding to hyperbolic space). The answer seems obvious to Poincaré: rather than consider space to be curved, scientists would find it "more advantageous" to suppose that starlight does not always propagate rectilinearly (Poincaré 1891 , 774). Poincaré implicitly assumes an alternative, non-Maxwellian optics to be feasible; he later extends the latter assumption to all of physics, by claiming that any experiment at all can be interpreted with respect to either Euclidean or hyperbolic space.

There are two aspects to Poincaré's conjecture I want to underline. First, scientists are free in Poincaré's scheme to choose between the two couples: Euclidean geometry and non-Maxwellian optics, or non-Euclidean geometry and Maxwellian optics. Either way, the geometry of space and the laws of optics result from a convention. In essentials, as Torretti $(1984,169)$ notes, Poincaré's view is equivalent to that of Helmholtz, to whom Poincaré refers his readers ${ }_{4}^{4}$ It also previews Pierre Duhem's holistic view of the structure of physical theory (Duhem 1906), which led Duhem to reject the possibility of crucial experiments.

Modern commentators like Zahar (2001, 100) understand Poincaré's conjecture to imply a "geometry plus physics" argument ranging over all possible geometries, including those of variable curvature, of the type Einstein employed in his general theory of relativity (1915). For Poincaré and others in the 1890s, however, geometries of constant curvature (i.e., Euclidean, hyperbolic, and spherical geometries) were the only plausible candidate geometries for physical space. The motivation for the latter restriction came from physics, which requires a theory of measurement. In the classical physics of Helmholtz and Poincaré, the act of measurement required free mobility of solids. In turn, free mobility of solids is possible only in Riemannian geometries of constant curvature. As we will see in $\S 4$, the motivation for relaxing the principle of free mobility of solids also came from physics: the physics of relativity.

In the second place, while Poincare recognized the freedom of scientists to choose a non-Euclidean geometry, he seems convinced

\footnotetext{
${ }^{4}$ Poincaré's notion of phenomenal space as an inseparable couple formed by geometry and physics was foreshadowed by Helmholtz's appeal to Lipschitz's argument in favor of a dynamics of hyperbolic space, based on the applicability of Hamilton's principle to spaces of nonzero constant curvature (Helmholtz 1995 238). On Helmholtz's empiricist philosophy of mathematics and geometry, see Volkert (1996) and Schiemann (1997). On Poincaré's reading of Helmholtz, see Heinzmann (2001).
} 
that they would never do so. Poincaré's overweening confidence in the convenience of Euclidean geometry for representing natural phenomena, come what may, separates him from most physicists and mathematicians of the late nineteenth century. It is often considered the weak link in Poincaré's philosophy of geometry. ${ }^{5}$

Poincaré presented a number of arguments in favor of the convenience of Euclidean geometry, none of which could be considered compelling. One putative advantage of Euclidean geometry is its simplicity, which Poincaré characterized algebraically (Torretti 1984 , 335). In the Euclid group, certain "displacements are interchangeable with one another, which is not true of the corresponding displacements of the Lobachevsky group" (Poincaré 1898b, 43). In other words, the Euclid group has a proper normal subgroup corresponding to translations, and according to this definition of simplicity, it is simpler than the hyperbolic group. Alternative criteria for simplicity abound, however, and Poincaré admitted quite freely that we would switch geometries, if confronted with a "considerably different" empirical base (Poincaré 1898b, 42). In sum, Poincaré's stance on the convenience of Euclidean geometry was no dogma. Rather, it reflected his great confidence in the future stability of the established baseline of experimental results, and in the explicative power of the principles of physics then in vigor.

Just what sort of experimental results might have led Poincaré to forgo Euclidean geometry at the close of the nineteenth century? Observations of stellar parallaxe could not have forced such a change, as we have seen. Poincaré did not elaborate; instead, he argued for the possibility of doing physics in hyperbolic space, which was an area of research little explored by nineteenth-century mathematicians (Walter 1999b, 92) $]^{6}$

Poincaré's view on the question of the equivalence of Euclidean and hyperbolic geometry is subject to debate. According to BenMenahem (2006, 41), Poincaré held all theorems of Euclidean geometry to have counterparts in hyperbolic geometry (and vice-versa). This equivalence in theorems would provide a template of sorts for the elaboration of a physics of hyperbolic space. Yet Poincaré never actually proposed such an equivalence (Torretti 1984, 336). Instead, in an attempt to characterize quadratic geometries, Poincaré wrote that in hyperbolic geometry we have a set of theorems "analogous" to those of Euclidean geometry (Poincaré 1887, 205).

What Poincaré offered in favor of his doctrine of space was a clever thought experiment, that builds on a suggestion made by Helmholtz. By wearing glasses fitted with convex lenses, Helmholtz (1995. 242) wrote, we can experience the optical effects of a world in which the natural geometry of space is non-Euclidean. Poincaré (1892b) deftly modified Helmholtz's example by imagining an apparently non-

\footnotetext{
${ }^{5}$ Poincaré's failure to convince scientists and philosophers to adopt his doctrine is noted by Torretti (1984, 256); his failure to convince mathematicians and physicists is shown by Walter (1997). For the characterization of the doctrine as a weak link, see Vuillemin (1972, 179) and Sklar (1974, 93).

${ }^{6}$ Exceptions include Eugenio Beltrami, Wilhelm Killing, and Rudolf Lipschitz, all of whom contributed to the mechanics of non-Euclidean space.
} 
Euclidean world, that of a heated sphere. In this way, Poincaré refocused his reader's attention away from intuition-a subject dear to Helmholtz-and toward a subject of his own predilection, the conventionality of laws of physics.

The heated sphere model of hyperbolic space fascinated Poincaré's contemporaries. Imagine a hollow sphere of radius $R$, heated in such a way that the absolute temperature at a point located a distance $r$ from the sphere's center is proportional to $R^{2}-r^{2} 7$ All bodies inside the sphere have the same coefficient of thermal dilation, and reach thermal equilibrium instantaneously. The atmosphere is such that the index of refraction is everywhere proportional to the reciprocal of temperature, and the trajectory of a light ray is described by an arc orthogonal to the enclosing sphere. The same arcs are materialized by the shortest distance between two points, as measured by a ruler, and the axis of rotation of a solid body. Poincaré suggests that the natives of such a world would adopt hyperbolic geometry for their measurements.

Highly contrived from a physical standpoint, Poincaré's model conveys quite well the idea that the adoption of Euclidean geometry is conditioned by certain features of our environment (such as the motion of solids). What is more, Poincaré $(1895,646)$ claimed that if physicists from planet Earth were transported to the heated world, they would continue to use Euclidean geometry, on the grounds that this would be the most convenient option available to them 8

The latter thesis is contested by Howard Stein (1987) on the basis of the extreme complexity of doing physics with Euclidean geometry in such a world. A terrestrial, pre-relativist physicist in the heated sphere would be led at first to posit a universal deforming force, only to find that the other laws she contrives conflict with those elaborated by physicists located in other regions of the world. Implicitly, Stein introduces a meta-theoretical commitment to a unified physics, and while Poincaré valued unified, interpenetrating explanations of physical phenomena, he recognized that such values are not imposed on us by the same phenomena. His doctrine of physical space affirms only that Earth-educated physicists can use Euclidean geometry inside the heated sphere, not that they must do so, or even that it is in their best interest to do so. As mentioned above, Poincaré readily admitted that physicists would forgo Euclidean geometry if the circumstances called for it. At the turn of the century, he was also quite sure that such circumstances would never arise.

\section{Poincaré's typology of hypotheses}

Poincaré's mature philosophy of science assigns a leading role to hypotheses. In the previous section, we recalled his belief that the axioms of geometry are conventions (or definitions in disguise), and

\footnotetext{
${ }^{7}$ For a rigorous discussion of the heated sphere's two-dimensional counterpart, the heated disk, see Barankin (1942).

${ }^{8}$ At first, Poincaré (1892b) maintained only that the sphere's natives would adopt non-Euclidean geometry.
} 
not hypotheses about the behavior of light rays or solid bodies. In this context, the question naturally arises of the relation between hypothesis and convention.

Hypotheses, according to Poincaré, are not all created equal. Some are more influential than others in determining the course of science, and Poincare found it useful to categorize the types of hypothesis he encountered according to their truth domain. An important impetus to this theorization of scientific hypotheses was provided by Heinrich Hertz's Principles of Mechanics (Lenard, ed. 1894), which made a singular impression on him, in virtue of its epistemic structure, and innovative use of hypothesis:

While the principles of dynamics have been exposed in many ways, the distinction between definition, experimental truth, and mathematical theorem has never been sufficient. This distinction is still not perfectly clear in the Hertzian system, and what is more, it introduces a fourth element: hypothesis. (Poincaré 1897, 743)

This fourth element corresponds to Hertz's assumption of hidden masses, which allowed him to forgo the concept of force. What strikes Poincaré above all is that Hertz's assumption is neither definition, nor experimental fact, nor theorem. It is what Poincaré would later call an "indifferent" hypothesis, in that an alternative hypothesis, or set of hypotheses, leads to the same result.

While Hertz's hypothesis of hidden masses was far too bold for Poincaré's taste, it inspired a new understanding of the role of hypothesis in physics, first unveiled at the international congress of physics held in Paris at the turn of the century. This was the first of two different typologies, which are now addressed in turn.

Poincaré $(1900,1166)$ identifies three sorts of hypotheses: generalizations, indifferent hypotheses, and natural hypotheses. The first sort is distinguished by the property of susceptibility to experimental corroboration. Once corroborated by experiment, these hypotheses become "fertile truths," without which there can be no increase in knowledge.

The second type of hypothesis, or "indifferent" hypothesis, serves to "fix our thought," but constitutes a non-unique premise in a deductive chain. Examples of indifferent hypotheses include the hidden masses of Hertz's mechanics, and the physical interpretation of the axial and polar vectors in classical optics. It can happen that a theorist prefers one hypothesis to another, to simplify a calculation, for instance, but experimental corroboration of the theory can have no bearing on the truth of the chosen hypothesis.

The third type of hypothesis is the "wholly natural" hypothesis. This vaguely-defined category concerns what might be described as experimental rules of thumb, without which measurement is nigh impossible. Natural hypotheses are accordingly the "last we ought to abandon." They include the law of continuity of cause and effect, and the vanishing force of very remote bodies. The latter hypothesis allows for multiple independent dynamical systems, as we shall see in $\S 4$. 
Poincaré's second typology of hypotheses appeared two years after the first, in the introduction to a collection of philosophical essays, La Science et l'hypothèse (Poincaré 1902). This revised typology features three categories, including the generalizations and indifferent hypotheses of the first typology, and excluding natural hypotheses. Poincaré may have folded natural hypotheses implicitly into the category of generalizations, since the former are, like the latter, accessible in principle to experiment. It is also possible, however, that neither typology was intended to be exhaustive, as suggested by Poincaré's decision to reprint his first typology in the ninth chapter of La Science et l'hypothèse.

A new type of hypothesis appears in Poincaré's 1902 typology: the "apparent" hypothesis. In fact, his third type of hypothesis is not a hypothesis at all, but a definition, or a "convention in disguise." Conventions, or apparent hypotheses, are essential to the activity of theorization in Poincaré's model of science.

For what follows, one final point needs to be underlined concerning the relation of Type I hypotheses (or hypotheses susceptible to experimental confirmation) to Type III hypotheses (or conventions). A physical law, if corroborated by experiment, can become a convention. Such a statutory evolution occurs when the law in question is deemed sufficiently fruitful to warrant protection from new experimental tests. Conventions are of great utility to the pursuit of scientific knowledge, but they are only definitions, and as such, may not be refuted by experiment. As an example, take the law of inertia, which Poincaré considers to be a convention. Any particular observation tending to disconfirm the law of inertia, Poincaré holds, would be dealt with by invoking the effect of invisible bodies, rather than by discarding the law of inertia (Poincaré 1902, 117).

\section{Relativity theory and the foundations of geometry}

On 5 June 1905, Poincaré presented a note to the French Academy of Sciences that put forth the foundations of the theory of relativity. It is in this short paper that Poincare expressed the Lorentz transformations in their modern form for the first time, along with the current density transformations (correcting Lorentz). In the 47-page memoir "On the dynamics of the electron" announced by this note, and that appeared in January 1906, Poincaré wrote the velocity transformations, characterized the Lie-algebra of the Lorentz group, and introduced a four-dimensional space in which three coordinate axes are real, and one is imaginary, inaugurating the era of four-dimensional physics. Building on Poincaré's ideas as well as those of Hertz, Lorentz, Einstein and Planck, the Göttingen mathematician Hermann Minkowski elaborated the theory of spacetime, which profoundly marked the philosophy of space and time, and was instrumental to Einstein's discovery of the general theory of relativity (Walter2007a).

Poincaré's contributions to the theory of relativity are well known 
to historians, but it is not entirely clear how Poincaré understood the theory of relativity to impinge upon his doctrine of space. In part, at least, this is Poincaré's doing, as he did not express himself clearly on this topic. As a result, commentators have offered a wide variety of interpretations of the relation between Poincaré's conventionalist philosophy and his discovery and interpretation of the theory of relativity. $9^{9}$

Although Poincaré does not comment in his 1906 memoir on the eventual influence of the principle of relativity on our conceptions of space and time, he points out an important consequence for the theory of measurement:

How do we go about measuring? The first response will be: we transport objects considered to be invariable solids, one on top of the other. But that is no longer true in the current theory if we admit the Lorentzian contraction. In this theory, two lengths are equal, by definition, if they are spanned by light in equal times. (Poincaré 1906, 132)

Poincaré spies a conflict between the traditional notion of rigidity and the principle of relativity, in that Lorentz-FitzGerald contraction appears to preclude the transport of rigid rulers upon which length measurement depends. Length congruence in Lorentz's theory depends not on the free mobility of invariable solids, but on the light standard.

Does this standard conflict necessarily with Poincaré's doctrine of space? Poincaré will provide an answer to the latter question, but only in the wake of spacetime theory, as discussed in the next section.

\section{The principle of physical relativity}

From 1905 to the end of his life (on 17 July 1912), Poincaré commented often on the theory of relativity, but only twice on the fourdimensional interpretation he had inaugurated. At first, he compared a possible four-dimensional language for physics to Hertz's mechanics, and observed that working out the corresponding formalism would entail "much pain for little profit" (Poincaré 1907). Poincaré's first and last words on the philosophical significance of spacetime were delivered on 4 May 1912, as the second in a series of four lectures at the University of London. His remarks were published posthumously as "L'espace et le temps," a title recalling that of Minkowski's celebrated 1908 lecture in Cologne, "Raum und Zeit" (Poincaré 1912; 1963, 97-109).

Although Poincaré might have derived satisfaction from the fact that Minkowski had based his spacetime theory on essentially the same four-dimensional geometry introduced in Poincaré's 1906 memoir, the Göttingen mathematician had noisily promoted an anti-conventionalist view of physical space and time, which was surely anathema to him (Walter 2010). Like Poincaré, Minkowski recognized that the

\footnotetext{
${ }^{9}$ For references, and an insightful comparison of Poincaré's and Einstein's philosophical approaches to relativity theory, see Paty (1993).
} 
new mechanics admitted no rigid bodies; unlike Poincaré, Minkowski presented the new intuitions (Anschauungen) of space and time not as conventions, but as the result of circumstances both empirical and formal that "forced themselves" upon scientists (Minkowski 1909, 79).

This is just the sort of anti-conventionalist view that Poincare targets in the opening of his London lecture on space and time:

Is not the principle of relativity, as Lorentz conceives it, going to impose an entirely new conception of space and time, and force us thereby to abandon conclusions that may have seemed established? (Poincaré 1963, 99)

Poincaré sees in Lorentz's principle of relativity a menace to his doctrine of physical space. But what does Poincaré take to be Lorentz's principle of relativity? He defines the latter in terms of group invariance:

The old form of the principle of relativity had to be abandoned; it is replaced by Lorentz's principle of relativity. The transformations of the "Lorentz group" are those that leave unaltered the differential equations of dynamics. (Poincaré 1963, 108)

According to Poincaré, Lorentz's principle of relativity is just Lorentz covariance, or what was then understood to be a succinct statement of the content of Einstein's special theory of relativity. Poincaré is poised to reconsider his brief observation of 1905 on the theory of measurement in Lorentz's theory, mentioned above ( $\S 3)$, and to show how his doctrine of physical space stands with respect to the theory of relativity.

Poincaré's views on how relativity theory interferes with classical concepts of space and time have significant historical interest, due to his foundational contributions to this theory. His London lecture on space and time represents his final word on this topic, and sets forth a fundamental change in his doctrine of physical space, by extending this doctrine to physical time. This extension is not well known, and is the focus of the following reconstruction of Poincaré's argument.

According to Poincaré, there is a principle of relativity, which he calls the "principle of physical relativity" (PPR), that exists in two forms. One of these is the Lorentz form, which Poincare refers to as Lorentz's principle of relativity. The other form is what I will refer to as the Galilei form, because it is defined by Galilei group invariance. The PPR holds that the differential equations by which we express physical laws

are altered neither by a change of fixed rectangular coordinate axes, nor by a change of temporal origin, nor by a substitution of mobile rectangular axes, the motion of which is a uniform, rectilinear translation. (Poincaré 1963, 102)

In modern terms, the PPR is equivalent to covariance with respect to a certain group of transformations. Poincaré distinguishes two groups 
in this context, corresponding to what were later known as the inhomogeneous Galilei transformations of the Galilei group, and the inhomogeneous Lorentz transformations of the Poincaré group.

The PPR has two main features, one of which is its testability. It is what Poincaré describes as an "experimental truth," i.e., a proposition susceptible to experimental disconfirmation. Recalling Poincaré's typology of hypotheses (see above, $\S 2$ ), the PPR is a Type I hypothesis. He expresses its empirical meaning in terms of two corollaries (Poincaré 1963, 106):

The reciprocal action of two bodies (or mechanical systems)

tends to zero as spatial separation increases indefinitely.

Two remote worlds behave as if independent.

Let us examine these corollaries one at a time. The first has a precedent in the 1900 lecture mentioned above, in which Poincaré first sought to characterize the sorts of hypotheses encountered in science. To hold that the "influence of very distant bodies is negligible," Poincaré wrote, was a "natural" hypothesis (Poincaré 1900, 1166). This natural hypothesis was announced some five years before the discovery of relativity theory, and twelve years before Poincaré updated his formula, ostensibly to accommodate the new physics of inertial frames.

The motivation for (1) remained the same over this twelve-year span. This particular natural hypothesis was designed to legislate away the effects of all long-range forces that fall off with increasing separation (such as gravitational and electromagnetic forces), and to create thereby the possibility of separate mechanical systems. As a consequence of what Poincaré calls the "principle of psychological relativity," recognizing the conventional nature of measurements of distance and duration, the existence of distant stars renders inertial frames of reference "purely conventional," obliging us, when we employ the concept of a frame of reference, to forgo "absolute rigor" (Poincaré 1963, 103).

The second corollary of the PPR has, like the first, a clear precedent in Poincaré's philosophy. In La Science et l'hypothèse, Poincaré analyzed the possibility of generalizing the Galilean principle of relative motion to include rotating frames of reference. If we want to solve a two-body problem based on Newton's Law, Poincaré remarked, we need to know the positions and velocities of the two gravitating bodies, as well as the corresponding initial values, along with "something else." It is this final missing bit of information that worried Poincaré, as Earman (1989, 86) observes. This solution element could be either the initial values of acceleration, or the area constant, or the absolute orientation of the universe, or the rate of change of orientation, or the position or velocity of Carl Neumann's Body Alpha. "We have," Poincaré wistfully concluded his earlier analysis, "but a choice of hypotheses" (Poincaré 1902, 137).

Poincaré's position advanced in this case, as well as for (1). His London lecture proposes a new argument in favor of (2), observing in essence, as Kerszberg $(1989,139)$ notes, that the missing solution 
element could be objectively determined, if only we disposed of not one universe, but of several universes. Poincaré imagines the situation as follows:

Instead of considering the entire universe, let's imagine small, separate worlds, visible to one another but free from outside mechanical action. If one of these worlds spins, we will see it spin, and recognize that the value to be assigned to the constant just mentioned depends on the spin velocity, and in this way, the convention habitually adopted by dynamicists will be justified. (Poincaré 1963, 105)

The PPR, Poincaré realizes here, provides a way out of his earlier dilemma, as it implies the existence of multiple independent mechanical systems in the universe, as expressed by (2).

Along with testability, a second salient feature of the PPR is its capacity to define space and time. The PPR "can serve to define space," by virtue of the fact that we perform measurements (or alternatively, in Poincaré's terminology, "construct space") by displacing solids and defining length congruence as coincidence of figures. The PPR admits the invariance under displacement of the form and dimensions of solids and other sufficiently-isolated mechanical systems, and thereby provides a foundation for length measurement. In Poincaré's words, the PPR provides us with a "new instrument of measurement" (Poincaré 1963, 106). Each possible displacement of a solid corresponds to a certain transformation, which leaves the form and dimensions of a given figure invariant. Such transformations, when taken together, form a group: the group of motions of invariable solids. While the principle of free mobility of solids selects any of three motion groups (Euclid, hyperbolic, spherical), the Galilei form of the PPR selects only one of these: the Euclid group. One imagines that Poincaré was uncomfortable with this particular consequence of the PPR, which he did not mention, but could not have ignored. Instead, he stressed the fact that, like the principle of free mobility of solids, the PPR provides a foundation for geometry ${ }^{10}$

Poincaré justified his replacement of the principle of free mobility of solids by the principle of form-invariance of the differential equations of mechanics in the following way. The motion group of invariable solids on one hand, and the symmetry group of mechanics on the other, give rise to conceptions of space that are not "essentially different" in Poincaré's view, because both groups define space in such a way that solids are unaltered in form when displaced. The role played by solid bodies in the old conception of the foundations of geometry goes over to the more general notion of a mechanical system. In fact, by defining space in terms of the motion group of solids, we affirm that the equations of equilibrium of solids do not vary upon displacement. In other words, we define space in such a way that the equilibrium equations of solids are unaltered by a change

\footnotetext{
${ }^{10}$ I differ here from Friedman $(2008,214)$, for whom "Poincaré's conception of space and geometry is entirely based [..] on the principle of free mobility first formulated by Helmholtz and then brought to precise mathematical fruition in the Helmholtz-Lie theorem."
} 
of axes. These equations of equilibrium are but a special case of the general equations of dynamics, Poincaré explains, and "according to the principle of physical relativity, they must not be modified by this change of axes." There is, consequently, no essential difference for Poincaré between the old way of defining space (postulating free mobility of solids), and the new one (postulating the symmetry group of mechanics), as far as solids are concerned.

Leaving solids behind, there are two significant differences between these two groups. The symmetry group of mechanics offers greater coverage than the motion group of solids, ranging over both solids and mechanical systems. This juxtaposition of the Euclid group and the symmetry group of mechanics was probably inspired by Minkowski's presentation of the theory of spacetime, which contrasted the Euclid and Galilei groups, although Poincaré neglected to mention Minkowski by name. ${ }^{11}$

The conception of space based on the symmetry group of mechanics differs from the one based on the Euclid group in a second respect, in that it

defines not only space, it defines time. It tells us the meaning of two simultaneous instants, of two equal times, or of a time twice as great as another. (Poincaré 1963, 107)

Both space and time are defined by the new view based on the symmetry group of mechanics, and this is significant for students of Poincaré's philosophy, as Paty (1996, 129) underlines, because he had never before admitted that the choice of an invariance group could define space and time. By preferring the symmetry group of mechanics to the motion group of solids, Poincaré considers the laws of mechanics to be more fundamental to our understanding of the world than the axioms of geometry. Put another way, Poincaré finds spacetime to be more fundamental than ordinary Euclidean space.

It may appear at this point that Poincaré has decided to renounce his doctrine of physical space in favor of the PPR. This is not so. The PPR, he explains, is an "experimental fact," and as such, it is "susceptible to incessant revision." The type of revision Poincaré has in mind implies a modification of the geometry of physical space. He was probably thinking of Einstein's program, announced in 1907, to generalize the principle of relativity to uniformly-accelerated frames of reference, and which led him to predict that rays of starlight must bend around the sun. What worries Poincaré is not so much a modification of the PPR as the consequential revision of the geometry of space. For the geometry of space to become immune to revision, the PPR must itself become immune to revision. In Poincaré's philosophical scheme, the only way to render an empirical law immune to revision is to promote it to conventional status. Naturally, this is what Poincaré decides to do, when he writes:

\footnotetext{
${ }^{11}$ Minkowski likewise neglected to mention Poincaré in his Cologne lecture, but acknowledged his contributions to relativity, along with those of Lorentz, Einstein, and Planck, in earlier papers (Walter 1999a).
} 
[Geometry] must become a convention again, [and] the principle of relativity must be considered as a convention. (Poincaré 1963, 107)

To drive this point home, Poincaré imagines a long-range force that diminishes at first with distance, then increases, producing motion inconsistent with the PPR's first empirical corollary (1). The PPR would then "appear to us as a convention" (le principe se présente à nous comme une convention, Poincaré 1963, 107), rather than as a Type I hypothesis, ostensibly because we would take measures to save the PPR from any experimental threat, by introducing a hidden mechanism, for example.

What Poincaré asks us to do, in other words, is to reconsider the epistemic status of the PPR, understood as an experimental, Type I hypothesis, and to promote it to a Type III, or apparent hypothesis (i.e., a convention). This promotion means that henceforth, the PPR is immune to empirical disconfirmation. It also means that the geometry of physical space can not be determined empirically, in complete compliance with Poincaré's doctrine of physical space.

Having explained how the doctrine of space may be salvaged by elevating the PPR to conventional status, Poincaré finally entertains a discussion of the Lorentz form of the PPR. The "recent progress in physics" has brought about a "revolution": Lorentz's principle has replaced the old one. To paraphrase in modern terms, Lorentz covariance has replaced Galilei covariance.

In the same way as the PPR with Galilei covariance can define space and time, the PPR with Lorentz covariance can define space and time:

It is as if time were a fourth dimension of space; and as if the four-dimensional space resulting from the combination of ordinary space and time could rotate not only about an ordinary space axis, in such a way that time is unaltered, but about any axis at all. To get a mathematically exact comparison we would have to assign purely imaginary values to this fourth space coordinate; the four coordinates of a point of our new space would not be $x, y, z$, and $t$, but $x, y, z$, and $t \sqrt{-1}$. (Poincaré 1963 . 108)

A four-dimensional vector space corresponding to the above description was introduced by Poincaré in the final section of his 1906 memoir on the dynamics of the electron, as a means of identifying Lorentzinvariant quantities to be used in a relativistic law of gravitational attraction. The new space he refers to in his London lecture, however, is not his own, but that of Minkowski, as Paty $(1996,132)$ observes. This much may be inferred from Poincaré's remark that in the new mechanics, and contrary to his earlier (pre-relativistic) analysis of simultaneity relations (Poincaré 1898a), there are events which can be neither the cause nor the effect of other given events. It was Minkowski who first identified such events, situated in a region of spacetime unique to what he called "spacelike" (raumartigen) vectors (Fig. 1). 


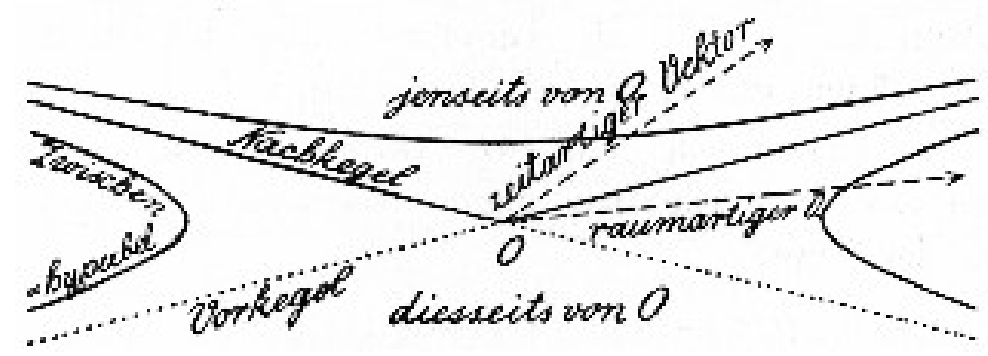

Figure 1: Lightcones in Minkowski spacetime (Minkowski 1909).

This insight was essentially tied to Minkowski's spacetime theory, providing apodictic proof of its fertility ${ }^{12}$

Poincaré says no more about Minkowski spacetime, which in 1912 was not yet well-known in Great Britain, but had already captured the attention of relativists in Germany and France, including Poincaré's former students Paul Langevin, a physicist at the Collège de France, and Émile Borel, a mathematician at the Sorbonne. He closes his lecture with the following words:

What will our stance be with respect to these new conceptions? Are we going to be forced to modify our conclusions? Of course not: we adopted a convention because it seemed convenient, and we said that nothing could oblige us to abandon it. Nowadays certain physicists want to adopt a new convention. It's not that they are obliged to do so, it's just that they judge the new convention to be more convenient. Those who feel differently may legitimately retain the old convention, so as not to disturb their habits. Between us, I believe this is what they will do for a good while longer. (Poincaré 1963,109$)$

In the wake of relativity theory, there is, as ever, no fact to the matter of the geometry of physical space, just a principle of (physical) relativity with a choice of invariance group. Poincare insists that his earlier conclusions need not be modified, and this is true, but in fact he has replaced a convention on space with a convention on spacetime.

The above-cited conclusion of Poincaré's London lecture on space and time raises a number of questions. First of all, does Poincaré's adoption of the PPR with Galilei covariance signal his disavowal of Lorentz covariance? Not at all: Poincaré distinguishes, as shown above, between the PPR as a Type I hypothesis, on one hand, and as a definition of spacetime, on the other. Undoubtedly, the same distinction applies if one associates the PPR with the Galilei group or the Lorentz group. By 1912, after some public hesitation, Poincaré had convinced himself of the experimental soundness of the new mechanics based on Lorentz covariance (Poincaré 1963, 110-111). The PPR with Lorentz covariance was then a viable candidate for elevation to conventional status. But instead of defining space and time in

\footnotetext{
${ }^{12}$ Minkowski $(1908, \S 6 ; 1909, \S$ III). Poincaré did not employ the Minkowskian term "spacetime" or any of its linguistic variants.
} 
virtue of Lorentz covariance, he prefers to define space and time in virtue of Galilei covariance.

Was such a position coherent at the time? Galilei and Lorentz conventions apply to the same inertial frames, and the quantities measured therein are either real or apparent, depending on the convention. Poincaré's interpretation of such quantities remained what we might call "apparentistic," in that the only true quantities were those of the ether frame. In principle, as Carl Neumann admitted in 1869, any inertial frame at all may be designated as the absolute (or ether) frame (Barbour 1989, 653), although Poincaré does not spell this out. Instead, he maintains that deformation of measuring devices due to motion with respect to absolute space can occur in such a way that this motion can never be detected (Poincaré 1963,99$){ }^{13}$ The latter proposition is itself a corollary of the PPR, although Poincaré does not present it as such.

From a superficial point of view, Poincaré's position may appear inconsistent, in that he postulates the PPR with Galilei covariance (or Galilei spacetime) while affirming the experimental validity of Lorentz covariance. If we recall his typology of hypotheses, however, the consistency of Poincaré's view is readily apparent. Considered as a Type I hypothesis, Lorentz covariance is an open question for experimenters. Consistency requires only that Lorentz-transformed space and time coordinates be interpreted with respect to the Galilei version of the PPR, the latter principle being understood as a Type III hypothesis. Poincaré satisfies this minimal requirement by referring to quantities measured in inertial frames as "apparent" quantities, with the "true" quantities belonging to the ether frame.

If we grant that Poincaré's defense of Galilei spacetime is both relativist and consistent, it may still appear convoluted, in that one could forgo the indirection of apparentism by adopting the Lorentz version of the PPR (or Minkowski spacetime). By doing so, quantities measured in inertial frames are "true," and the concept of ether is rendered wholly superfluous. I suspect, however, that in 1912 Poincaré's position appeared less convoluted to most physicists than the latter one, due to the conceptual inertia of three centuries of uncritical acceptance of absolute space and time.

The PPR with Lorentz covariance, attributed by Poincaré to "certain physicists," defines space and time in terms of a four-dimensional Minkowski spacetime geometry. The leading proponents of Minkowski's spacetime theory in 1912 included Poincaré's correspondent Arnold Sommerfeld, Minkowski's former students Max Born, Max von Laue, Gunnar Nordström, and Theodor Kaluza, Minkowski's former colleagues Max Abraham and Gustav Herglotz, the Greifswald physicist Gustav Mie, and the mathematical physicist Philipp Frank in Vienna. One has to wonder about Poincaré's characterization of the spacetime theorists' understanding of spacetime. Did these theo-

\footnotetext{
${ }^{13}$ For a reevaluation of Poincaré's dynamic approach to relativity, see Brown (2005). Although Brown claims Poincaré never recognized time dilation (Brown 2005, 147), this effect appears to have been no less real for him than that of length contraction. See, for example, Poincaré's remarks on "The new mechanics," delivered in Lille on 2 August 1909 (Poincaré 1909. 173).
} 
rists consider Minkowski spacetime to be a convention in Poincaré's sense? If this were so, Lorentz covariance could no longer have been a subject of experiment for them. In fact, well before Poincaré's speech, and well after, several physicists and astronomers were engaged in verifying consequences of the theory of relativity. Consequently, Poincaré's attribution of a conventional view of space and time to spacetime theorists is probably best understood as wishful thinking.

Einstein, like most physicists at the time, understood Lorentz covariance as a hypothesis subject to experimental corroboration. In a letter to his friend Friedrich Adler, a Machian anti-relativist imprisoned for the cold-blooded assassination of the Prime Minister of the Austro-Hungarian Empire, Einstein denied that Lorentz covariance was conventional. Referring to the Lorentz transformations in the form

$$
x^{\prime}=\ell \beta(x-u t), y^{\prime}=\ell y, z^{\prime}=\ell z, t^{\prime}=\ell \beta\left(t-\frac{u}{c^{2}} x\right)
$$

where $\beta=1 / \sqrt{1-u^{2} / c^{2}}$, Einstein focused his argument on the nature of the constant $\ell$ :

It is clear in any event that the choice of $\ell$ implies no mere formal convention, but a hypothesis characterizing reality. [...] Thus Bucherer, for example, backed a theory for a while, which comes out of a different choice of $\ell$. Nowadays there is no further question of a different choice of $\ell$, since the electron's laws of motion have been verified with increased precision. (Einstein to F. Adler, 29.09.1918, Schulmann et al.1998, Doc. 628)

Einstein does not refer to Poincaré in his letter to Adler, but contemporary correspondence shows that he was familiar with the Frenchman's philosophy of science. The cited passage expresses concern over the epistemological status of Lorentz covariance, which Einstein considers to be a well-verified physical hypothesis, and not a mere definition or convention.14

\section{Why Galilei spacetime?}

The two principal approaches to special relativity in 1912, associated, on one hand, with Lorentz and Poincaré, and on the other hand, with Einstein and Minkowski, may be distinguished by their respective ontologies, but also by their performance. We've seen that Poincaré considered two forms of the principle of physical relativity: a Galilean form, defining Galilei spacetime, and a Lorentzian form,

\footnotetext{
${ }^{14} \mathrm{~A}$ few days before writing to Adler, Einstein agreed with the German mathematician Eduard Study's criticism of Poincaré's doctrine of space; see Einstein to E. Study, 25.09.1918 (Einstein 1998, Doc. 624). But a few months later, Einstein remarked to the philosopher Hans Vaihinger that Poincaré's view of the role of Euclidean geometry in science was "wesentlich tiefer" than that of Study (Einstein to Vaihinger, 03.05.1919, in Saß 1979, 319). On Vaihinger and Poincaré, see the contribution by Christophe Bouriau in the present volume (Bouriau 2009).
} 
defining Minkowski spacetime. We've also seen that Poincaré preferred one form to the other. Since his reasons for preferring Galilei spacetime to Minkowski spacetime are not readily discernible in his London lecture on space and time, we are prompted to look elsewhere in order to understand his view.

The first historian to hazard an explanation for Poincaré's choice was Gerald Holton, who sought to explain Poincaré's attachment to the concept of ether as a consequence of his conservative outlook on science, and described the French mathematician as the "most brilliant conservator of his day" (Holton 1973, 189). Holton seems not to have known that Poincaré was in his day one of the best-known critics of the foundations of physics, alongside Ernst Mach and Heinrich Hertz. Poincaré's fellow scientists considered him to be the most lucid of theoretical physicists, on the leading edge of the latest discoveries (Walter 2007b). In Émile Borel's opinion, for example, Poincaré "contributed more than anyone to the creation of what may be called the spirit of twentieth-century theories of physics, as opposed to those of the nineteenth century" (Borel 1924). In light of such views, it seems impossible to explain Poincaré's attachment to the ether - or Galilei spacetime - as the result of a conservative tendency.

Recent studies of Poincaré's scientific activity suggest a quite different way of understanding his preference for Galilei spacetime. A top graduate of Paris's elite, state-run engineering schools, Poincaré was more skilled than most in the practical arts of civil and mechanical engineering. From the 1890s on, he contributed to engineering journals, taught electrical engineering, and urged fellow scientists to "increase the output of the scientific machine," as Galison (2003, 201) aptly notes. In almost identical terms, Poincaré described the scientific role of theoretical physics, whose duty it was to "guide generalization in such a way as to increase the output [...] of science" (Poincaré 1900, 1164).

Polytechnicians like Poincaré were trained to identify the options most likely to enhance productivity, and while Poincaré certainly had several evaluative criteria at hand when comparing Galilei and Minkowski spacetime, that of scientific productivity was likely to have been a leading candidate. From any reasonable standpoint, looking back in time from 1912, the Galilei convention had as much to recommend it as the Lorentz convention, since both approaches could claim relativistic theories of the electron, mechanics, electrodynamics of moving media, and gravitation.

Certain facts of life, however, would have argued against the Galilei convention. For instance, the publishing trend in theoretical relativity favored Minkowski spacetime (Walter 1999b), and the brightest young German and French theorists were either convinced Minkowskians, or were, like Einstein, soon to adopt a Minkowskian spacetime ontology. Poincaré was undoubtedly aware of these facts, and his lecture in London can be read as an effort to stem the tide of Minkowskian relativity. It turned out to be his final effort, as his life ended ten weeks later. He was a skilled and perceptive critic on scientific matters, and one can't help but speculate upon how he would have 
reacted to two signal developments that took place the year after his death. Of the two events I have in mind, one would appear to underline the cogency of Poincaré's preference for Galilei spacetime, and the other, its drawbacks.

The limitations of the Galilei form of the PPR are most obvious when Einstein's general theory of relativity is taken into account, the first elaborate expression of which was Einstein and Großmann's Entwurf theory (Einstein \& Großmann 1913). Like almost all of his contemporaries, Poincaré was unprepared for a theory of spacetime as a four-dimensional, pseudo-Riemannian manifold with curvature determined by the distribution of matter and energy. In a sense, the ultimate success of Einstein's theory was also that of the Lorentz form of the PPR, in that Einstein expected Lorentz covariance to hold in the limiting case of weak gravitational fields. At the time of Poincaré's London lecture, however, the secular advance of Mercury's perihelion was an anomaly under both the Galilei and Lorentz forms of the PPR, as Poincaré was the first to point out (Walter 2007a, 208).

The second development is that of Niels Bohr's first model of the atom, which discarded a central tenet of classical physics by confining the electron in a hydrogen atom to one of a discrete set of circular orbits, in ordinary space and time. Arnold Sommerfeld altered the model in 1915-1916 to allow for elliptical orbits precessing relativistically, and found an explanation for the fine structure of the spectral lines of hydrogen. In effect, Sommerfeld extended the reach of relativistic dynamics to the inner regions of the hydrogen atom. As a bonus of sorts, the sophisticated analytical methods developed by Poincaré for celestial mechanics in the 1890s soon found application in a modified version of the Bohr atom (Darrigol 1992, chap. 6).

There is ample reason to believe that Poincaré was prepared for a theory such as Bohr's. In the fall of 1911, he participated in discussions of the theory of quanta as a member of the First Solvay Council, along with Einstein, Planck, Sommerfeld, Nernst and others. As Staley (2005) observes, Poincaré was struck by the fact that these physicists already referred to relativistic mechanics as the "old mechanics," the new mechanics being that of energy quanta. Shortly after the Solvay Council, Poincaré showed (as did Paul Ehrenfest) the quantum hypothesis to be necessary and sufficient for the establishment of Planck's law (Prentis 1995). Once Poincaré recognized the mechanics of quanta to be incompatible with both ordinary and relativistic mechanics (Poincaré 1963, 111, 125), he had all the more reason to emphasize the conventional nature of both Galilei and Minkowski spacetime.

The principle of physical relativity, which encodes a spacetime view of physical phenomena, was largely ignored in Poincaré's time, while his basic insight later informed a great number of investigations in theoretical and mathematical physics. Poincaré's early study of the role of hypothesis in the physical sciences, inspired in part by his reading of Hertz's mechanics (as shown in $\S 2$ ), served as an essential resource for his elaboration of the principle of physical relativity, and exemplifies the close intertwining of philosophical reflection 
and physical understanding at the forefront of research in the natural sciences.

\section{Acknowledgments}

My thanks go to Yemima Ben-Menahem, Olivier Darrigol, Arthur Fine, Gerhard Heinzmann, Albert Martínez, Erhard Scholz, and the editors for discussion and insightful comments.

\section{References}

Barankin, E. W. Heat flow and non-Euclidean geometry. American Mathematical Monthly 49(1) (1942): 4-14.

Barbour, J. B. Absolute or Relative Motion? Vol. 1: The Discovery of Dynamics. Cambridge: Cambridge University Press, 1989.

Ben-Menahem, Y. Conventionalism: From Poincaré to Quine. Cambridge: Cambridge University Press, 2006.

Borel, E. Henri Poincaré. Revue scientifique 62(11) (1924): 321-324.

Bouriau, C. Vaihinger and Poincaré: an original pragmatism. In The Significance of the Hypothetical in the Natural Sciences. Edited by M. Heidelberger and G. Schiemann, 221-249. Berlin: Walter de Gruyter, 2009.

Brown, H. R. Physical Relativity: Space-Time Structure from a Dynamical Perspective. Oxford: Oxford University Press, 2005.

Darrigol, O. From c-Numbers to q-Numbers: The Classical Analogy in the History of Quantum Theory. Berkeley: University of California Press, 1992.

Duhem, P. La Théorie physique: son objet et sa structure. Paris: Chevalier \& Rivière, 1906.

Earman, J. World Enough and Space-Time: Absolute vs. Relational Theories of Space and Time. Cambridge MA: MIT Press, 1989.

Einstein, A. and Großmann, M. Entwurf einer verallgemeinerten Relativitätstheorie und einer Theorie der Gravitation. Leipzig: Teubner, 1913.

Friedman, M. L. Space, time, and geometry: Einstein and Logical Empiricism. In Einstein for the 21st Century. Edited by P. L. Galison, G. Holton, and S. S. Schweber, 205-216. Princeton: Princeton University Press, 2008.

Galison, P. Einstein's Clocks and Poincaré's Maps: Empires of Time. New York: Norton, 2003.

Gray, J. and Walter, S., eds. Henri Poincaré: Trois suppléments sur la découverte des fonctions fuchsiennes. Berlin: Akademie-Verlag, 1997a. 
Gray, J. and Walter, S. Introduction. In Gray and Walter (1997a), $1-25,1997 b$.

Heinzmann, G. The foundations of geometry and the concept of motion: Helmholtz and Poincaré. Science in Context 14 (2001): 457470.

Helmholtz, H. v. On the origin and the significance of geometrical axioms. In Science and Culture: Popular and Philosophical Essays by Hermann von Helmholtz. Edited by D. Cahan, 226-248. Chicago: University of Chicago Press, 1995.

Holton, G. Thematic Origins of Scientific Thought: Kepler to Einstein. Cambridge MA: Harvard University Press, 1973.

Kerszberg, P. The Invented Universe: The Einstein-De Sitter Controversy (1916-17) and the Rise of Relativistic Cosmology. Oxford: Oxford University Press, 1989.

Lenard, P., ed. Gesammelte Werke von H. Hertz, Bd. 3: Die Prinzipien der Mechanik in neuem Zussamenhänge dargestellt. Leipzig: J. A. Barth, 1894.

Minkowski, H. Die Grundgleichungen für die electromagnetischen Vorgänge in bewegten Körpern. Nachrichten von der Königlichen Gesellschaft der Wissenschaften zu Göttingen (1908): 53-111.

-. Raum und Zeit. Jahresbericht der deutschen MathematikerVereinigung 18 (1909): 75-88.

Nabonnand, P. La polémique entre Poincaré et Russell au sujet du statut des axiomes de la géométrie. Revue d'histoire des mathématiques 6 (2000): 219-269.

Panza, M. L'intuition et l'évidence; la philosophie kantienne et les géométries non euclidiennes : relecture d'une discussion. In Les savants et l'épistémologie vers la fin du XIX siècle. Edited by M. Panza and J.-C. Pont, 39-87. Paris: Blanchard, 1995.

Paty, M. Einstein philosophe : la physique comme pratique philosophique. Paris: Presses Universitaires de France, 1993.

-. Poincaré et le principe de relativité. In Henri Poincaré : Science et philosophie. Edited by J.-L. Greffe, G. Heinzmann, and K. Lorenz, 101-143. Berlin: Akademie Verlag, 1996.

Poincaré, H. Sur les hypothèses fondamentales de la géométrie. Bulletin de la Société Mathématique de France 15 (1887): 203-216.

-. Les géométries non euclidiennes. Revue générale des sciences pures et appliquées 2 (1891): 769-774.

—. Non-Euclidian geometry. Nature 45 (1892a): 404-407.

—. Sur les géométries non euclidiennes. Revue générale des sciences pures et appliquées 3 (1892b): 74-75. 
—. L'espace et la géométrie. Revue de métaphysique et de morale 3 (1895): 631-646.

-. Les idées de Hertz sur la mécanique. Revue générale des sciences pures et appliquées 8 (1897): 734-743.

- La mesure du temps. Revue de métaphysique et de morale 6 (1898a): 1-13.

-. On the foundations of geometry. Monist 9 (1898b): 1-43.

- Les relations entre la physique expérimentale et la physique mathématique. Revue générale des sciences pures et appliquées 11 (1900): 1163-1175.

—. La science et l'hypothèse. Paris: Flammarion, 1902.

- L'espace et ses trois dimensions. Revue de métaphysique et de morale 11 (1903): 281-301, 407-429.

-. Sur la dynamique de l'électron. Rendiconti del Circolo Matematico di Palermo 21 (1906): 129-176.

—. La relativité de l'espace. Année psychologique 13 (1907): 1-17.

—. La mécanique nouvelle. Revue scientifique 12 (1909): 170-177.

-. L'espace et le temps. Scientia (Rivista di Scienza) 12 (1912): 159-170.

—. Dernières pensées. Paris: Flammarion, 2d edition, 1963.

—. Premier supplément. In Gray and Walter (1997a), 27-73, 1997.

Pont, J.-C. L'aventure des parallèles : histoire de la géométrie non euclidienne; précurseurs et attardés. Bern: Peter Lang, 1986.

Prentis, J. J. Poincaré's proof of the quantum discontinuity of nature. American Journal of Physics 63(4) (1995): 339-350.

Rollet, L. Henri Poincaré, des mathématiques à la philosophie : étude du parcours intellectuel, social et politique d'un mathématicien au début du siècle. Lille: Éditions du Septentrion, 2001.

Saß, H.-M. Einstein über „wahre Kultur“ und die Stellung der Geometrie im Wissenschaftssystem. Zeitschrift für allgemeine Wissenschaftstheorie 10 (1979): 316-319.

Schiemann, G. Wahrheitsgewissheitsverlust: Hermann von Helmholtz' Mechanismus im Anbruch der Moderne. Darmstadt: Wissenschaftliche Buchgesellschaft, 1997.

Schulmann, R., Kox, A. J., Janssen, M., and Illy, J., eds. The Collected Papers of Albert Einstein, Volume 8, The Berlin Years: Correspondence, 1914-1918. 2 vols. Princeton: Princeton University Press, 1998. 
Sklar, L. Space, Time and Spacetime. Berkeley: University of California Press, 1974.

Staley, R. On the co-creation of classical and modern physics. Isis 96(4) (2005): 530-558.

Stein, H. After the Baltimore Lectures: some philosophical reflections on the subsequent development of physics. In Kelvin's Baltimore Lectures and Modern Theoretical Physics: Historical and Philosophical Perspectives. Edited by R. Kargon and P. Achinstein, 375-398. Cambridge, MA: MIT Press, 1987.

Torretti, R. Philosophy of Geometry from Riemann to Poincaré. Dordrecht: Reidel, 2d edition, 1984.

Volkert, K. Hermann von Helmholtz und die Grundlagen der Geometrie. In Hermann von Helmholtz: Vorträge eines Heidelberger Symposiums anläßlich des einhundertsten Todestages. Edited by W. U. Eckart and K. Volkert, 177-205. Pfaffenweiler: Centaurus, 1996.

Vuillemin, J. Poincaré's philosophy of space. Synthese 24 (1972): 161-179.

Walter, S. A. La vérité en géométrie : sur le rejet mathématique de la doctrine conventionnaliste. Philosophia Scientia 2 (1997): 103135 .

- Minkowski, mathematicians, and the mathematical theory of relativity. In The Expanding Worlds of General Relativity. Edited by H. Goenner, J. Renn, T. Sauer, and J. Ritter, 45-86. Einstein Studies 7. Boston/Basel: Birkhäuser, 1999a.

- The non-Euclidean style of Minkowskian relativity. In The Symbolic Universe: Geometry and Physics, 1890-1930. Edited by J. Gray, 91-127. Oxford: Oxford University Press, 1999b.

-. Breaking in the 4-vectors: the four-dimensional movement in gravitation, 1905-1910. In The Genesis of General Relativity, Volume 3. Edited by J. Renn and M. Schemmel, 193-252. Berlin: Springer, 2007a.

-. Introduction. In La correspondance d'Henri Poincaré, Volume 2: La correspondance entre Henri Poincaré et les physiciens, chimistes et ingénieurs. Edited by S. Walter, ix-xvi. Basel: Birkhäuser, 2007b.

-. Minkowski's modern world. In Minkowski Spacetime: A Hundred Years Later. Edited by V. Petkov, 43-61. Fundamental Theories of Physics 165. Berlin: Springer, 2010.

Zahar, E. Poincaré's philosophy of geometry, or does geometric conventionalism deserve its name? Studies in History and Philosophy of Modern Physics 28 (1997): 183-218. 
-. Poincaré's Philosophy from Conventionalism to Phenomenology. Chicago: Open Court, 2001. 